\title{
RELACIONES INTERINSTITUCIONALES PARA LA SALUD CENTRADA EN LA PERSONA
}

\author{
Miguel R. Jorge $\mathrm{e}^{1,2}$
}

\begin{abstract}
RESUMEN
Desde 2005, cuando la Asociación Mundial de Psiquiatría presentó su Programa Institucional de Psiquiatría para la Persona, muchas organizaciones están trabajando en conjunto para que los cuidados médicos sean menos orientados a las enfermedades y mas centrados en la persona como un todo. Hoy en día, más que 30 organizaciones están relacionadas en promover la medicina centrada en la persona, donde se destacan algunas de sus iniciativas.
\end{abstract}

Palabras Clave: salud; medicina; Salud holística; Organizaciones (fuente: DECS BIREME).

\section{INTERINSTITUTIONAL RELATIONS FOR PERSON-CENTERED HEALTH}

\begin{abstract}
Since 2005, when the World Psychiatric Association has launched its Institutional Program on Psychiatry for the Person, many different organizations have worked together to advance medical care from a disease-oriented challenge to a focus centered in the whole person. Nowadays, over 30 organizations are related in promoting person-centered medicine, from which some initiatives are highlighted.
\end{abstract}

Key Words: Health; Medicine; Holistic Health; Organizations (Source: MeSH NLM).

Diferentes organizaciones relacionadas con la salud alrededor del mundo apuntan a la persona como centro de atención, en lugar de enfocarse en las enfermedades. La definición de1946 de la Organización Mundial de la Salud, considera a la salud como un estado de bienestar completo, tanto físico como mental y social y no únicamente como la ausencia de enfermedad y dolencias ${ }^{(1)}$.

Más recientemente, la Asamblea Mundial de la Salud de 2008, órgano de la Organización Mundial de la Salud (WHO, por sus siglas en inglés), aprobó una resolución destinada a renovar el cuidado de salud primaria, la cual incluye [...] reorientar el cuidado de salud convencional hacia la gente que requiere cuidados primarios ${ }^{(2)}$.

La Asociación Médica Mundial (WMA, por sus siglas en inglés) ${ }^{(3)}$ es una federación global conformada por 112 asociaciones médicas nacionales (NMAs, por sus siglas en inglés), la cual representa a más de 9 millones de médicos de diferentes regiones del mundo. Está orientada al logro de los mayores estándares posibles de cuidado médico, ética, educación y derechos relacionados a la salud humana para todo individuo. Su dependencia más importante lo constituye la Asamblea General, donde todas las NMAs están representadas. Adicionalmente, la WMA cuenta con un Consejo que incluye a más de 20 NMAs, representantes de médicos, representantes de diferentes regiones del mundo y de las NMAs. Los miembros del Consejo son asignados a tres Comités (Ética Médica, Asuntos médico-sociales, y Financiamiento y Planificación) y un Comité Ejecutivo que dirige el trabajo día a día de la Asociación.

Uno de los principales objetivos de la WMA es crear políticas dirigidas a guiar las actividades de los médicos a través del globo. Estas políticas incluyen diferentes tipos de documentos: resoluciones específicas, limitadas a región o tiempo; declaraciones referidas a problemas globales, y declaraciones relacionadas a preguntas/cuestionamientos fundamentales. Hay tres

\footnotetext{
Universidad Federal de Sao Paulo. Sao Paulo, Brasil.

2 Asociación Médica Mundial (WMA). Ferney-Voltaire, Francia.

Presentado en las “Jornadas Latinoamericanas de Medicina Centrada en la Persona”, Lima, Perú, 18-19 diciembre, 2015.

Recibido: 21/09/2016 Aprobado: 26/10/2016
}

Citar como: Jorge MR. Relaciones interinstitucionales para la salud centrada en la persona. Rev Peru Med Exp Salud Publica. 2016;33(4):824-6. doi: 10.17843/ rpmesp.2016.334.2571 
documentos de gran importancia para los miembros constituyentes de la WMA: la Declaración de Ginebra, el Código Internacional de Ética Médica y la Declaración de Helsinki. La Declaración de Ginebra ${ }^{(4)}$ instituye a alguien que va a ser médico: La salud de mi paciente será mi consideración más importante...; el Código de Ética Médica Internacional (5) afirma: Cuando un médico ofrece atención médica deberá actuar en el mejor interés del paciente...; y la Declaración de Helsinki ${ }^{(6)}$ establece: Es deber del médico promover y salvaguardar la salud, bienestar y derechos de los pacientes, inclusive de aquellos que están involucrados en una investigación médica... En la actualidad, estos documentos, usualmente, emplean el término "paciente", cuando se refieren a gente, pero cuando consideramos el contexto, es claro que la persona total es quien está en el centro de la atención del médico.

En el año 2005, la Asociación Psiquiátrica Mundial (WPA, por sus siglas en inglés) - bajo la presidencia de Juan Enrique Mezzich - estableció un Programa Institucional de Psiquiatría para la Persona (IPPP, por sus siglas en inglés), articulando ciencia y humanismo y "afirmando a la persona entera/completa en su contexto como el centro y meta del cuidado clínico y la promoción de salud, tanto a nivel individual como de comunidad" (7).

En el transcurso de los siguientes años, Mezzich y sus colaboradores/asociados iniciaron relaciones con otras organizaciones internacionales médicas y de salud, iniciativa que llevó a la preparación de la Primera Conferencia de Ginebra sobre la Medicina Centrada en la Persona (IPPP, por sus siglas en inglés), en 2008. En ese primer momento, muchas organizaciones estuvieron presentes, participando en las actividades de la conferencia, tales como WPA, IPPP, la WMA, la Organización de Doctores de Familia/ Médicos de Cabecera (WONCA, por sus siglas en inglés), la Federación Mundial de Neurología (WFN, por sus siglas en inglés), la Federación Mundial de Educación Médica (WFME, por sus siglas en inglés), el Consejo para Organizaciones Internacionales de Ciencias Médicas (CIOMS, por sus siglas en inglés), la Federación Mundial de Salud Mental (WFMH, por sus siglas en inglés), el Consejo Internacional de Enfermeras (ICN, por sus siglas en inglés), la Alianza Internacional de Organizaciones de Pacientes (IAPO, por sus siglas en inglés), y la Sociedad Paul Tournier ${ }^{(7)}$. Durante la Segunda Conferencia en 2009, los participantes discutieron el desarrollo de una Red Internacional de Medicina Centrada en la Persona
(INPCM, por sus siglas en inglés), la cual fue seguida por el Colegio Internacional de Medicina Centrada en la Persona (ICPCM, por sus siglas en inglés) ${ }^{(8)}$.

Las Conferencias de Ginebra se convirtieron en un modelo de colaboración interinstitucional ya que [...] han recibido el coauspicio de un considerable número de entidades internacionales, y han crecido gradualmente desde 10 en su primera edición hasta por encima de 30 en las últimas. Este último número comprende... un número de organizaciones profesionales, federaciones de familias, colegios médicos, institutos de investigación $y$ universidades ${ }^{(9)}$.

A pesar de todo esto, la Medicina Centrada en la Persona aún no ha logrado ser ampliamente adoptada en los currículos médicos y extendida a la práctica clínica cotidiana. Según la Federación Mundial de Educación Médica (WFME, por sus siglas en inglés), los siguientes aspectos contribuyen a la situación actual: impacto insuficiente en la ética médica, insuficiente énfasis en habilidades de comunicación, insuficiente entrenamiento en psicología y psiquiatría médicas, insuficiente educación en sociología médica y disciplinas de salud pública, reducción en el entrenamiento general y especialización demasiado precoz y consecuencias de algunos realineamientos en el sector de cuidado de la salud con influencia negativa en el ambiente educacional ${ }^{(10)}$.

Teóricamente, el concepto de menor tecnología y mayor humanismo y un acercamiento holístico en el cuidado médico están difundidos y bien aceptados, pero - en general - la realidad de los sistemas de salud a través del mundo nos muestra una imagen diferente: la historia médica, enfocada en los síntomas, se lleva a cabo en pocos minutos, se solicitan muchas pruebas de laboratorio e, incluso, procedimientos caros como tomografías computarizadas, resonancias magnéticas ${ }^{(1)}$, énfasis en la identificación y tratamiento de enfermedades sin tomar en consideración los aspectos personales y sociales del contexto, y manejo terapéutico restringido a prescripción de drogas. En muchos países, particularmente en los menos desarrollados, la figura se empeora por una enorme diferencia entre la atención médica privada y la pública.

La iniciativa de la Medicina Centrada en la Persona y el representativo número de organizaciones involucradas es aún una promesa de hacer una diferencia para un mejor cuidado de salud. 
1. World Health Organization (WHO). New York: WHO; 1946 [cited 19 Jul 2016]. Available from: http://www. who.int/about/definition/en/print. html

2. World Health Organization (WHO). Primary health care: now more than ever [Internet]. Geneva: WHO; 2008 [cited 19 Jul 2016]. World Health Report 2008. Available from: http:// www.who.int/whr/2008/en/

3. World Medical Association (WMA). Geneva: WMA, 2016 [cited 19 Jul 2016]. Available from: www.wma.net

4. World Medical Association (WMA). Declaration of Geneva [Internet]. Geneva: WMA; 1948 [updated May 2006; cited 19 Jul 2016]. Available from: http://www.wma.net/ en/30publications/10policies/g1/ index.html
5. World Medical Association (WMA). International Code of Medical Ethics [Internet]. London: WMA; 1949 [updated October 2006; cited 19 Jul 2016]. Available from: http://www.wma.net/ en/30publications/ 10 policies/c8/ index.html

6. World Medical Association (WMA). Declaration of Helsinki [Internet]. Helsinki: WMA; 1964 [updated October 2013; cited 19 Jul 2016]. Available from: http://www.wma.net/ en/30publications/10policies/b3/ index.html

7. Mezzich JE. World Psychiatric Association perspectives on personcentered psychiatry and medicine. Int J Integr Care. 2010; 10(5): 3-7.

8. Mezzich JE. The construction of person-centered medicine and the launching of an International College. Int J Pers Cent Med. 2012; 2(1): 6-10.

9. Mezzich JE, Appleyard J, Botbol $M$, et al. International journey and the development of person centered medicine. Int J Pers Cent Med. 2014; 4(4): 211-6.

10. Karle H. World Federation for Medical Education perspectives on personcentered medicine. Int J Integr Care. 2010; 10(5): 14-17.

Correspondencia: Miguel Roberto Jorge Dirección: Rua Manoel da Nobrega 801 Casa 17, CEP )4001-084, Sao Paulo-SP, Brasil

Teléfono: +5511 99183-5162

Correo electrónico:miguelrjorge@gmail.com

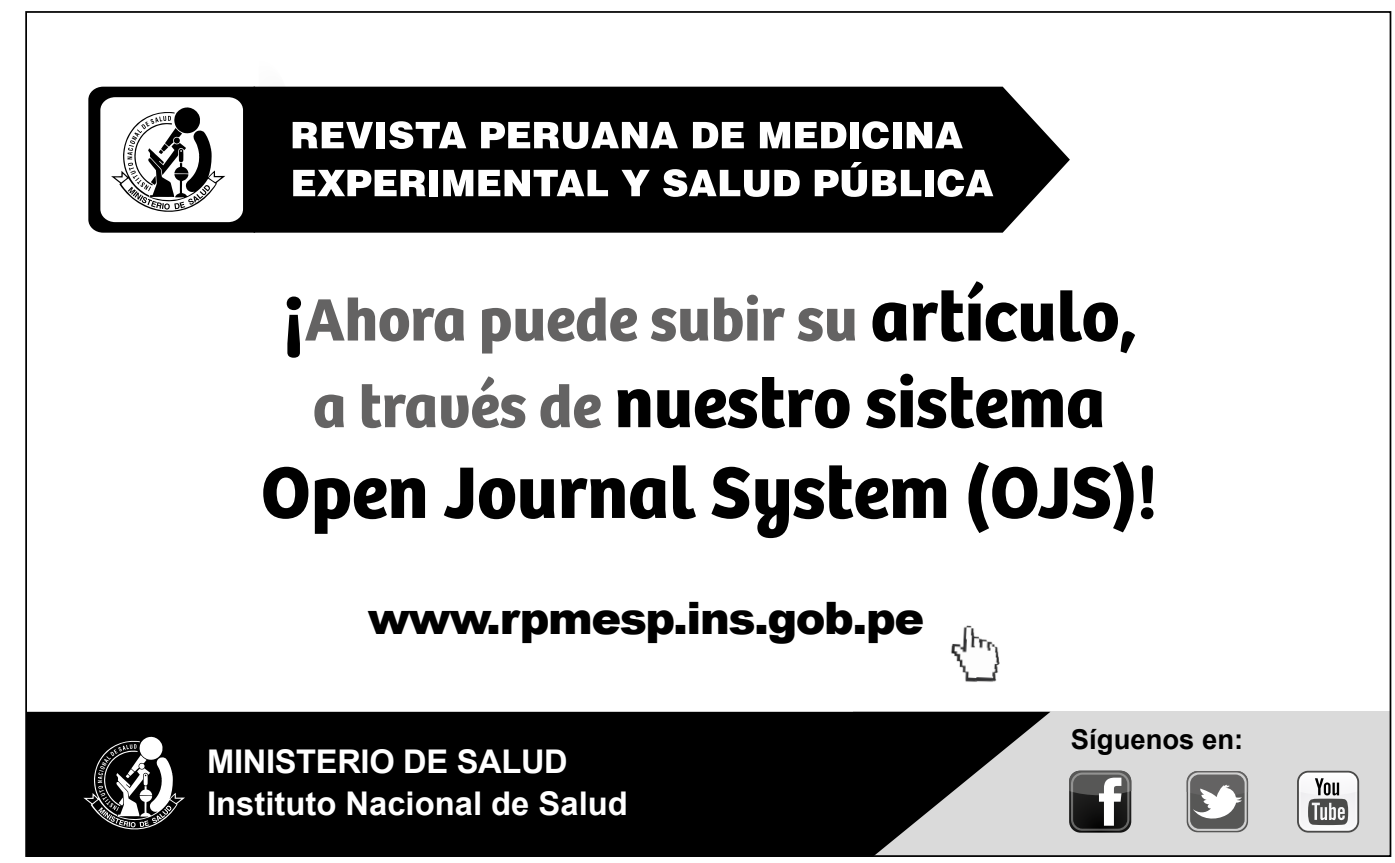

